\title{
Accuracy of coronary computed tomography angiography for bioresorbable scaffold luminal investigation: a comparison with optical coherence tomography
}

\author{
Carlos Collet $^{1} \cdot$ Yohei Sotomi $^{1} \cdot$ Rafael Cavalcante $^{2} \cdot$ Taku Asano $^{1} \cdot$ Yosuke Miyazaki $^{2} \cdot$ Erhan Tenekecioglu $^{2} \cdot$ \\ Pieter Kistlaar $^{3,4} \cdot$ Yaping Zeng $^{2} \cdot$ Pannipa Suwanasson $^{5} \cdot$ Robbert J. de Winter $^{1} \cdot$ Koen Nieman $^{2}$ • \\ Patrick W. Serruys ${ }^{6} \cdot$ Yoshinobu Onuma $^{2,7}$
}

Received: 14 September 2016 / Accepted: 8 November 2016 / Published online: 28 November 2016

(C) Springer Science+Business Media Dordrecht 2016

\begin{abstract}
To establish the accuracy of coronary computed tomography angiography (CTA) for in-scaffold quantitative evaluation with optical coherence tomography (OCT) as a reference. The translucent backbone of the bioresorbable scaffold allow us to evaluate non-invasively the coronary lumen with coronary CTA. In the ABSORB first-inman studies, coronary CTA was shown to be feasible for quantitative luminal assessment. Nevertheless, a comparison with an intravascular modality with higher resolution has never been performed. In the ABSORB Cohort B trial, 101 patient with non-complex lesions were treated with the fully biodegradable vascular scaffold. For this analysis, all patients who underwent coronary CTA at 18 months and OCT within \pm 180 days were included. Coronary CTA and OCT data were analysed at an independent core laboratory for quantitative cross-sectional luminal dimensions. The primary objective was the accuracy and precision of
\end{abstract}

Clinical Trial Registration-URL: http://www.clinicaltrials.gov. Unique identifier: NCT00856856.

Patrick W. Serruys

patrick.w.j.c.serruys@pwserruys.com

1 Cardiology Department, Academic Medical Centre, Amsterdam, The Netherlands

2 ThoraxCenter, Erasmus University Medical Centre, Rotterdam, The Netherlands

3 Division of Image Processing, Department of Radiology, Leiden University, Leiden, The Netherlands

4 Medis medical imaging systems BV, Leiden, The Netherlands

5 Northern Region Heart Center, Faculty of Medicine, Chiang Mai University, Chiang Mai, Thailand

6 Imperial College of London, London, UK

7 Cardialysis BV, Rotterdam, The Netherlands coronary CTA for in-scaffold minimal lumen area assessment, with OCT as a reference. Among the 101 patients of the ABSORB Cohort B trial, 35 underwent both OCT and coronary CTA. The feasibility of quantitative evaluation was $74 \%$. In the scaffolded segment, coronary CTA underestimated minimal lumen area by $9.8 \%$ (accuracy $0.39 \mathrm{~mm}^{2}$, precision $1.0 \mathrm{~mm}^{2}, 95 \%$ limits of agreement -1.71 to $2.50 \mathrm{~mm}^{2}$ ). A similar level of agreement was observed in the non-scaffolded segment. Compared to OCT, coronary CTA appears to be accurate for the estimation of in-scaffold luminal areas, with no difference compared to the non-scaffolded region.

Keywords Coronary computed tomography angiography $\cdot$ Polymeric scaffolds · Optical coherence tomography

$\begin{array}{ll}\text { Abbreviations } \\ \text { BVS } & \text { Bioresobable vascular scaffold } \\ \text { CTA } & \text { Computed tomography angiography } \\ \text { IVUS } & \text { Intravascular ultrasound } \\ \text { FFR }_{\text {CT }} & \begin{array}{l}\text { Fractional flow reserve derived from computed } \\ \text { tomography }\end{array} \\ \text { OCT } & \text { Optical coherence tomography } \\ \text { PCI } & \text { Percutaneous coronary interventions } \\ \text { PLLA } & \text { Poly-L-lactide }\end{array}$

\section{Introduction}

The field of interventional cardiology has been revolutionized by the introduction of the Absorb bioresorbable vascular scaffolds (Absorb BVS, Abbott Vascular, Santa Clara, $\mathrm{CA})$. The restoration of the vascular integrity of the vessel might supersede the long-term limitations seen with 
permanent metallic stents [1]. At mid-term follow-up, randomised trials have shown comparable clinical results between bioresorbable vascular scaffolds (BVS) and cobaltchromium everolimus-eluting stent for the treatment of non-complex coronary lesions [2, 3]. Also, the translucent backbone of the BVS allows for the non-invasive investigation of the coronary lumen with coronary CTA, thereby overcoming the limitations observed in patients previously revascularized with metallic stents [4].

In the ABSORB first-in-man trials (Cohort A and B), serial coronary CTA was performed. In these studies, high feasibility for quantitative in-scaffold luminal assessment was reported. In addition, the functional component of the treated region was assessed using computational fluid dynamics. Fractional flow reserve derived from coronary CTA (i.e., FFR ${ }_{\mathrm{CT}}$ ) was serially assessed. The persistence of $\mathrm{FFR}_{\mathrm{CT}}$ normalization at long-term follow-up was observed [4]. The accuracy and precision of luminal geometry segmentation are of paramount importance for both quantitative luminal analysis and $\mathrm{FFR}_{\mathrm{CT}}$ computation.

Over the past two decades, major advances have been introduced in the field of computed tomography (i.e., the advent of 256 and 320-detectors scanners, dual-source CT, iterative algorithm for reconstructions, etc). With the best-in-class CT scanners available, the spatial resolution has been reported in the range of 250 microns. In contrast, invasive optical coherence tomography (OCT) has a resolution of 10-20 microns and is widely recognized as a gold standard for coronary luminal measurements [5]. Nevertheless, a comparison of in-scaffold luminal areas between coronary CTA and an intravascular modality with higher resolution have never been performed.

Therefore, the aim of this study was: (1) To assess the accuracy of coronary CTA for in-scaffold quantitative luminal analysis at mid-term follow-up with OCT as a reference and; (2) To validate the coronary CTA-derived luminal area measurement at the level of the radiopaque marker.

\section{Methods}

\section{Study design}

In the ABSORB B (ABSORB Clinical Investigation Cohort B, Everolimus Eluting Coronary Stent System Clinical Investigation) trial, one hundred and one patients with non-complex de novo coronary lesion were enrolled at 12 centres between March and November 2009. Informed consent was obtained from all individual participants included in the study. The details of the trial have been previously described elsewhere [6]. Seventy-one patients underwent coronary CTA at 18 months with the use of at least a 64-detector (or higher) CT scanner [7]. In addition, patients were divided into two groups (B1 and B2) for serial invasive imaging (e.g., angiography, intravascular ultrasound and OCT) at different time points [6]. OCT was performed at 12 or 24-month follow-up in 28 and 31 patients in cohort B1 and B2, respectively [8]. All patients with coronary CTA and OCT evaluations were included in the present analysis (Fig. 1).

\section{Study device and procedure}

The BVS balloon-expandable device consists of a polymer backbone of poly-L-lactide (PLLA) coated with a thin layer of a 1:1 mixture of poly(L-lactide-co-D, L-lactide) (PDLLA) polymer and the antiproliferative drug everolimus containing $100 \mu \mathrm{g}$ everolimus $/ \mathrm{cm}^{2}$ scaffold. All patients included in this study received a bioresorbable scaffold of $3 \mathrm{~mm}$ in diameter and $18 \mathrm{~mm}$ in length. The implant is radiolucent but has two platinum markers of $244 \mu \mathrm{m}$ at each end that allows for easy visualisation on CTA [9]. Both PLLA and PDLLA are fully resorbable [10].

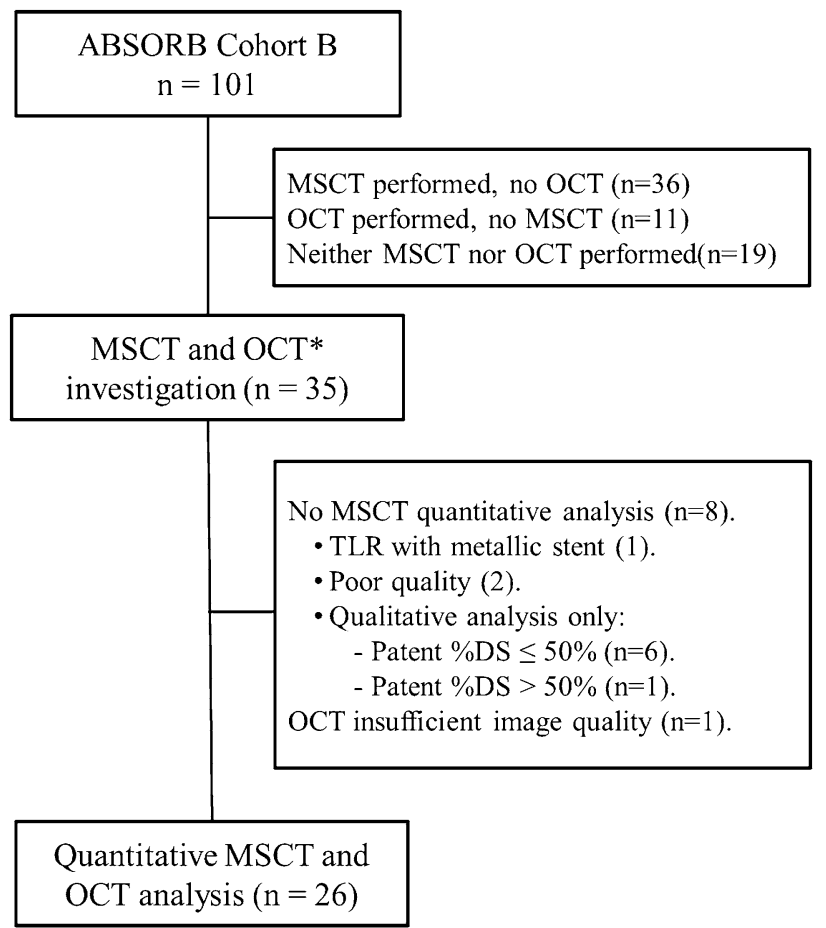

Fig. 1 Flowchart of patients included in this analysis. From the overall ABSORB Cohort B cohort $(n=101), 35$ patients underwent both coronary CTA and OCT analysis. Eight cases were non-analyzable. For this analysis 26 patients with both image modalities were included. CTA computed tomography angiography, TLR Target vessel revascularization, $O C T$ Optical coherence tomography 


\section{Coronary CTA Acquisition}

Coronary CTA angiography was performed with different CT scanners: GE Lightspeed VCT 64-detector [10], Phillips Brilliance 64-detector [4], Siemens Flash 256-detector [15], Phillip Brilliance ICT 256-detector [6]. Standard acquisition techniques were used, which included betablockers in patients with heart rate $>65 \mathrm{bpm}$, tube settings depending on patient body mass index $(80-140 \mathrm{kV})$, and axial scan protocols for patients with lower heart rates to reduce radiation doses, all at the discretion of the individual sites [11]. Images were reconstructed using thin slices $(0.5-0.67 \mathrm{~mm})$ and medium smooth reconstruction filters in different phases. All data were stored on a DVD for core laboratory evaluation.

\section{Coronary CTA analysis}

Data from coronary CTA was analysed off-line by an independent Corelab (Cardialysis BV, Rotterdam, The Netherlands) using a validated cardiovascular analysis package (QAngioCT Research Edition version 3.0.14, Medis, Leiden, The Netherland). Vessel cross-sections were reconstructed at approximately $0.5 \mathrm{~mm}$ longitudinal increments, using the platinum scaffold markers as landmarks. Automatic lumen segmentation of the vessel lumen was performed; manual corrections using an attraction points tool was allowed. A window display setting of level 750 Hounsfield units and width 250 Hounsfield units were used for the analysis, and was adjusted if necessary. In addition, gradient images were used to assist the detection of the luminal contour. The minimal lumen area (MLA) was determined for each scaffold. The non-scaffolded lumen area was assessed $5 \mathrm{~mm}$ proximal and distal to each marker. To evaluate the concordance in the axial distribution of the MLA, each scaffold of $18 \mathrm{~mm}$ length was divided into three segments. The position of the scaffold MLA was measured from the distal radiopaque marker and compared from the distance obtained from OCT. Concordance was defined as localization of the MLA in the same scaffold segments (i.e., proximal, mid and distal). Additionally, to investigate the lumen contour tracing method at the level of the marker crosssection, three strategies were compared based on the agreement with the OCT-derived lumen area. The marker was divided in three parts by 2 parallel lines. Afterward, the extrapolation of the luminal contour through the marker artefact was manually drawn tangential to the first line i.e., closest to the lumen (first strategy), to the centre of the marker (second strategy) and to the second line (third strategy). The gradient image visualization tool was used to identify the centre of the marker.

\section{Optical coherence tomography analysis}

OCT acquisitions were performed using three different commercially available systems: the M2 and M3 Time- Domain Systems and the C7XR Fourier-Domain System (LightLab Imaging, Westford, Massachusetts). OCT images were acquired at frame rates of 15.6, 20, and $100 \mathrm{frames} / \mathrm{s}$ with pullback speeds of 2,3 , and $20 \mathrm{~mm} / \mathrm{s}$ in the M2Time-Domain System,M3 Time- Domain System, and C7XR Fourier-Domain System (LightLab Imaging), respectively. All recordings were performed according to the recommended procedure for each OCT system [12]. The OCT measurements were performed with the QCUCMS software version 4.69 (Leiden University Medical Center, Leiden, The Netherlands) by the core laboratory (Cardialysis BV, Rotterdam, the Netherlands). The following parameters were evaluated: MLA, lumen area at the proximal and distal marker cross-section, and the distance from the distal marker to the MLA. For the proximal and distal reference segments, the luminal area at the non-scaffolded region was measured at $5 \mathrm{~mm}$ from the proximal and distal radiopaque markers. Bifurcation segments in which the side branch occupied more than $45^{\circ}$ of the cross-section were excluded in order to avoid tracing interpolation when quantifying the lumen [13]. In case the metallic marker of the scaffold could not be identified due to the wire shadow artefact or insufficient flush of blood, the cross-section and the associated proximal or distal edge cross-sections were not included in the analysis.

The primary objective was to assess the accuracy of coronary CTA for the measurement of in-scaffold minimal lumen area with OCT as a reference. The secondary objective was to validate the strategy of coronary CTA luminal contour tracing at the level of the radiopaque markers with matched OCT area as a reference.

\section{Statistical analysis}

Binary variables are presented as percentages and continuous variables as mean and standard deviation or median and interquartile range, as appropriate. Continuous variables with normal distributions were compared using $t$ test. The level of agreement between methods at the cross-section level was determined using the Bland-Altman method and the Passing-Bablok regression analysis [14]. For the Bland-Altman method, data are given as plots showing the absolute difference between corresponding measurements of both methods (y-axis) against the average of both methods (x-axis). Accuracy was defined as the mean difference between OCT and coronary CTA and precision as the standard deviation of the difference. The $95 \%$ limits of agreement were calculated as mean bias $\pm 1.96 \times$ standard deviation. For the Passing-Bablok regression analysis, the 
$\alpha$ and $\beta$ coefficient assess for the systematic and proportional differences. If 0 is in the confident interval (CI) of $\alpha$, and 1 is in the $\mathrm{CI}$ of $\beta$, the two methods are comparable. If 0 is not in the CI of $\alpha$ there is a systematic difference and if 1 is not in the $\mathrm{CI}$ of $\beta$ then there is a proportional difference between the two methods. Intra and inter-observer variability were assessed using intra-class correlation coefficients. All statistical analyses were performed with SPSS software version 23.0 (IBM, Armonk, New York). This study was funded by Abbott Vascular.

\section{Results}

Overall, 35 patients with both OCT and coronary CTA evaluations were included in this analysis. Baseline clinical and lesions characteristics are shown in Table 1. The mean age was $63.2 \pm 9.0$ years and $77 \%$ were male. The Left Anterior Descending artery was the most frequent treated vessel in $46 \%$ of the cases. Lesions were classified according to AHA/ACC as B1 and B2 in $90 \%$ of the cases. Images were acquired with a mean difference of $182 \pm 19$ days. CTA images were acquired with a mean heart rate of $53.8 \pm 6.82 \mathrm{bpm}$. Coronary CTA quantitative analysis in the scaffold region was possible in 26 cases (76\%) (Fig. 1). There were no systematic or proportional differences in minimum luminal area assessment between OCT and coronary CTA $\left(4.48 \pm 1.57 \mathrm{~mm}^{2}\right.$ OCT versus $4.04 \pm 1.35 \mathrm{~mm}^{2}$

Table 1 Baseline characteristics

\begin{tabular}{ll}
\hline & Overall $(\mathrm{n}=35)$ \\
\hline Age (mean \pm SD), years & $63.2 \pm 9.0$ \\
Male sex, $\mathrm{n}(\%)$ & $27(77)$ \\
Diabetes mellitus, $\mathrm{n}(\%)$ & $2(3)$ \\
Current smoker $\mathrm{n}(\%)$ & $9(25)$ \\
Hyperlipidemia requiring medication, $\mathrm{n}(\%)$ & $27(77)$ \\
Hypertension, $\mathrm{n}(\%)$ & $21(60)$ \\
Prior PCTA, $\mathrm{n}(\%)$ & $7(20)$ \\
Prior myocardial infarction, $\mathrm{n}(\%)$ & $10(29)$ \\
Treated vessel & \\
Left anterior descending, $\mathrm{n}(\%)$ & $16(46)$ \\
Left circumflex, $\mathrm{n}(\%)$ & $8(23)$ \\
Right coronary artery, $\mathrm{n}(\%)$ & $11(31)$ \\
AHA/ACC lesion classification & \\
A, $\mathrm{n}(\%)$ & $1(3)$ \\
B1, $\mathrm{n}(\%)$ & $17(48)$ \\
B2, $\mathrm{n}(\%)$ & $15(42)$ \\
C, $\mathrm{n}(\%)$ & $2(6)$ \\
Mean reference vessel diameter, (mm) & $2.53 \pm 0.57$ \\
Minimum luminal diameter, $(\mathrm{mm})$ & $1.05 \pm 0.29$ \\
Diameter stenosis, $(\%)$ & $56 \pm 13.7$ \\
\hline
\end{tabular}

coronary CTA, $\alpha$ coefficient $-1.1295 \%$ CI -4.78 to 0.66 , $\beta$ coefficient $1.1295 \%$ CI 0.70 to 1.9$)$ respectively. Coronary CTA underestimated luminal area by $9.8 \%$ in both scaffolded and non-scaffolded segments. In the scaffolded region, the accuracy and precision of coronary CTA at the MLA was $0.39 \pm 1.0 \mathrm{~mm}^{2}$ (95\% limits of agreements -1.71 to $2.50 \mathrm{~mm}^{2}$ ). In addition, the analysis of 31 matched crosssections in the non-scaffolded segments at the proximal and distal edge showed similar level of agreement compared to the scaffolded region (accuracy $0.45 \pm 0.70 \mathrm{~mm}^{2}$, limits of agreement -0.92 to $1.82 \mathrm{~mm}^{2}$, $\alpha$ coefficient $-0.01395 \% \mathrm{CI}$ -0.9 to $0.86, \beta$ coefficient $0.9395 \%$ CI $0.75-1.08$ ) (Fig. 2). Concordance of axial in-scaffold MLA localization was observed in $77 \%$ of the cases (Case example Fig. 3). The intra-class correlation coefficient of in-scaffold MLA measurements for inter-observer agreement was 0.98 (95\% CI $0.93-0.99 ; \mathrm{p}<0.001)$ and 0.98 (95\% CI $0.94-0.99)$ for intra-observer agreement.

\section{Luminal assessment at the level of the radiopaque marker}

Forty cross sections at the level of the radiopaque marker were evaluated (16 were excluded due to impossibility to identify the marker using OCT $(n=13)$ and overlap with a metallic stent $(n=3))$. When the luminal contour was traced across the centre of the radiopaque marker (i.e., second strategy) the coronary CTA-derived lumen area showed a mean difference of the $0.48 \mathrm{~mm}^{2}$ (limits of agreements -1.55 to $2.51 \mathrm{~mm}^{2}$ ) with OCT-derived matched area as a reference. The inclusion in the luminal segmentation of one-third (first strategy) and two-thirds (third strategy) showed a mean difference of $1.21 \mathrm{~mm}^{2}$ (limits of agreements -0.68 to $3.11 \mathrm{~mm}^{2}$ ), and $-0.58 \mathrm{~mm}^{2}$ (limits of agreements -2.95 to $1.74 \mathrm{~mm}^{2}$ ), respectively (Fig. 4). Figure 5 shows the overall agreement in MLA and marker cross-section luminal areas between OCT and coronary CTA.

\section{Discussion}

The main findings of this analysis are (1) At 18 months, coronary CTA-derived in-scaffold minimal lumen area showed good agreement with OCT-derived minimal lumen area; (2) At the level of the marker with the blooming artefact, luminal segmentation through the centre of the marker showed the best agreement with the matched OCT-derived area; and (3) There was similar agreement between luminal measurement at the scaffolded and non-scaffolded segments.

Non-invasive coronary CTA have demonstrated high accuracy for the evaluation of native coronary vessels 

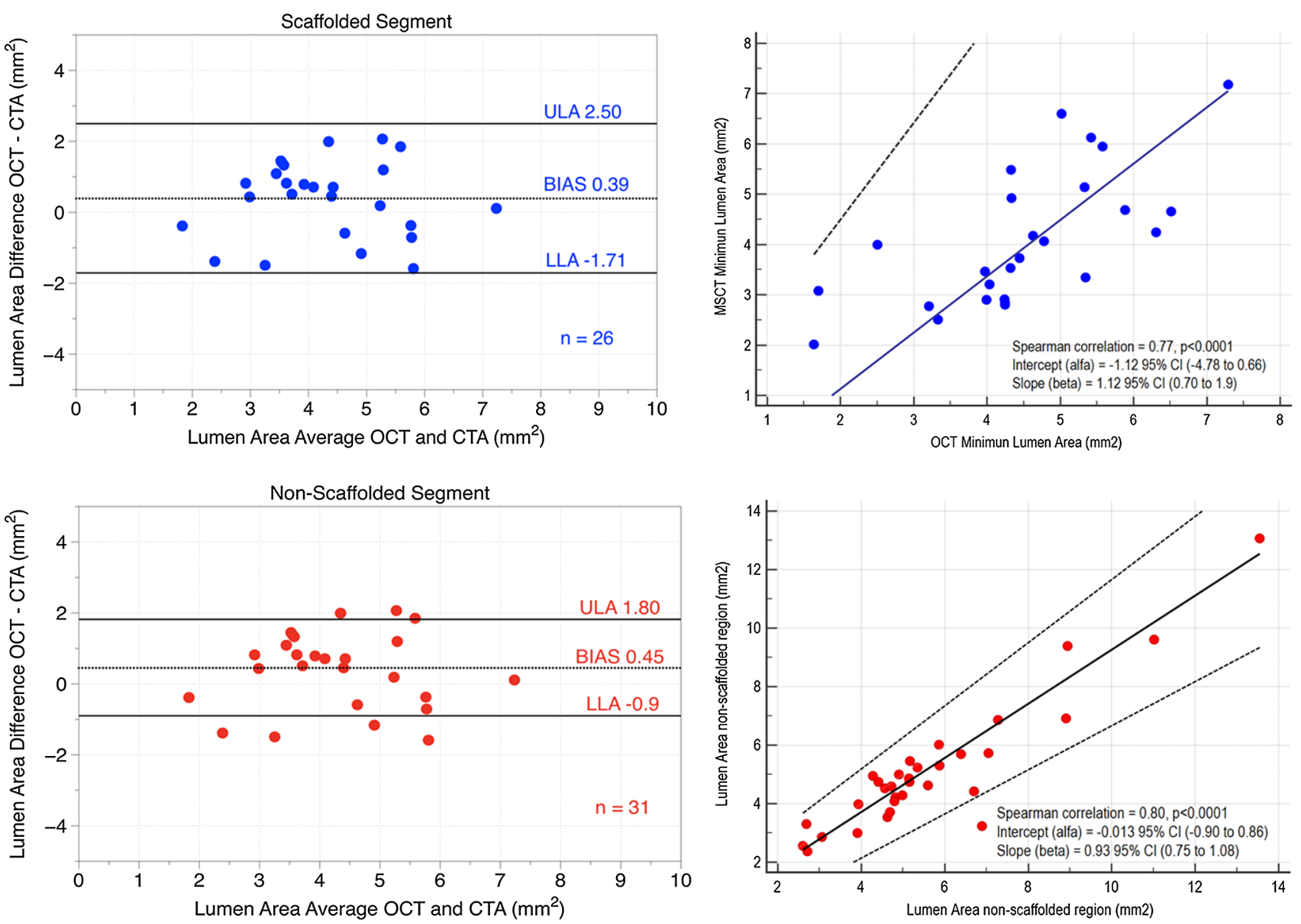

Fig. 2 (Left side) Bland-Altman plots of the mean differences in luminal areas between optical coherence tomography CTA in scaffolded (top) and non-scaffolded segments (bottom). In the right side

compared to invasive coronary angiography. In the landmark multicentre CORE-64 study, coronary CTA showed high diagnostic accuracy for the detection of coronary lesions with diameter stenosis $\geq 50 \%$ [15]. Moreover, a meta-analysis assessing the accuracy of coronary CTA to measure coronary luminal area at the cross-section level found an excellent agreement (mean difference of $0.23 \mathrm{~mm}^{2}$, CI $95 \%-0.07$ to 0.48 ) compared to IVUS [16]. In this study, coronary CTA underestimated in-scaffold MLA by $0.39 \mathrm{~mm}^{2}$ compared to OCT $\left(4.48 \pm 1.57 \mathrm{~mm}^{2}\right.$ OCT versus $4.04 \pm 1.35 \mathrm{~mm}^{2} \mathrm{CTA}$ ). Similar agreement was observed in the non-scaffolded region (mean difference $0.45 \mathrm{~mm}^{2}$, limits of agreement -0.92 to $1.82 \mathrm{~mm}^{2}$ ). These findings are comparable with previous reports comparing luminal areas derived from IVUS and coronary CTA in native coronary vessels [16].

Coronary CTA evaluation in segments treated with metallic stents is hampered by the presence of the metal; whereas the polymeric struts of the biodegradable scaffold do not interfere with non-invasive luminal evaluation of the scatterplots with passing-Bablok analysis between modalities in both scaffolded (top) and non-scaffolded segments (bottom)

[17]. Moreover, high concordance in axial MLA localization $(77 \%)$ was observed. In three of the six cases with discordant MLA localization between modalities, the MLA derived from the OCT was at the level of the radiopaque marker. At this level, the blooming artefact from the radiopaque marker might have interfered with an appropriate luminal evaluation by coronary CTA; therefore, contributing to the relocation of the MLA.

The focal partial volume averaging and bean hardening artefacts at the scaffold marker cross-section makes coronary luminal segmentation challenging. Two 244 micron platinum markers at each site of scaffold create a blooming artefact leading to an artificial increase in the size of the structure that precludes appropriate visualization of the lumen boundary. At the marker cross-section, we reported the agreement between three coronary CTA luminal segmentation tracing strategies. The inclusion of the centre of the marker in the luminal area was associated with the higher accuracy $\left(0.48 \mathrm{~mm}^{2}, 95 \%\right.$ limits of agreements -1.55 to $2.51 \mathrm{~mm}^{2}$ ). This is technically relevant, since 


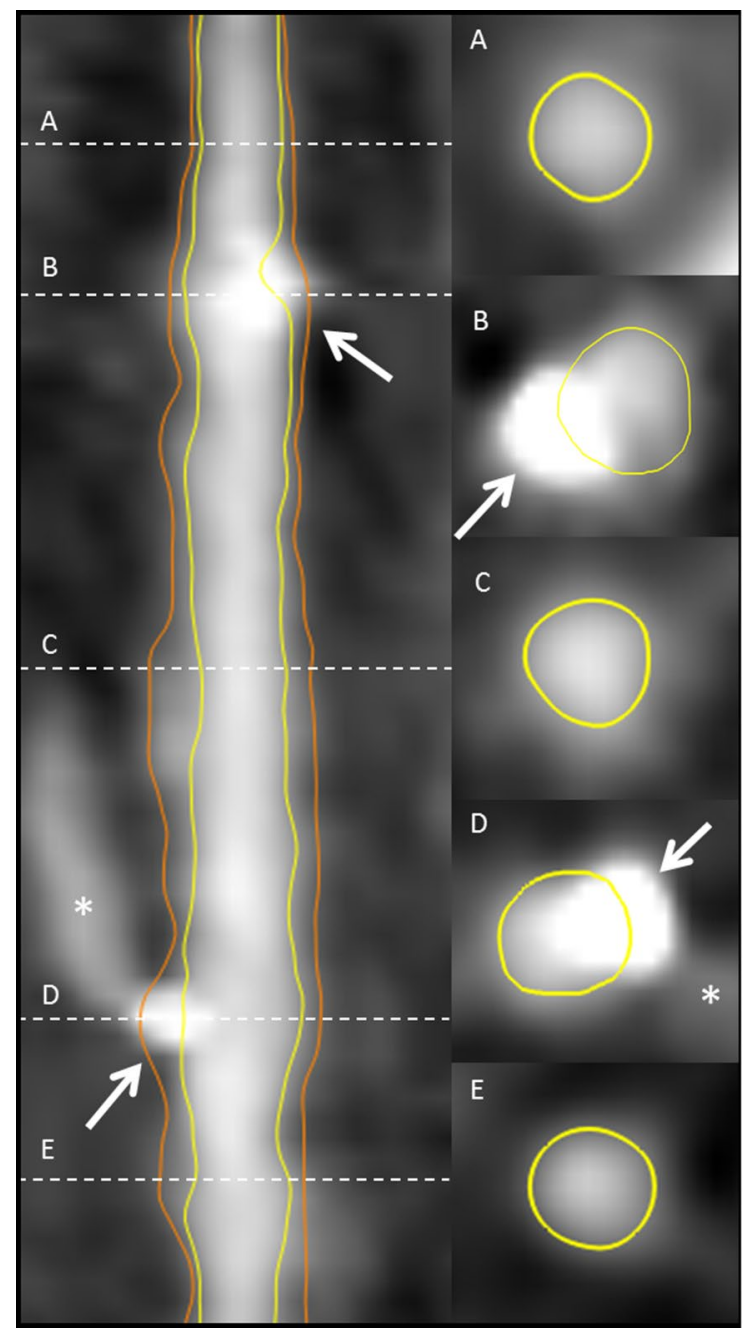

Fig. 3 Case example of an coronary CTA straight multiplanar reconstruction image and optical coherence tomography correlating matched luminal areas at the scaffold and non-scaffolded segments. Panels a, $\mathbf{a}^{\prime}$ and $\mathbf{e}, \mathbf{e}^{\prime}$ show the vessel lumen at the non-scaffolded segments. Panels $\mathbf{b}, \mathbf{b}^{\prime}$ and $\mathbf{d}, \mathbf{d}^{\prime}$ show the cross-section at the level of

automated luminal contour detection algorithms might recognized the blooming artefact of the marker with high Hounsfield units (mean $1435 \pm 412 \mathrm{HU}$ ) as dense calcium and exclude it from the lumen creating a systematic underestimation of the luminal area at the marker cross-section. Therefore, manual correction of the luminal contour in needed at this level. The accuracy of in-scaffold quantitative luminal analysis observed in this study supports the use of coronary CTA as the modality of choice for the follow-up of patients treated with bioresorbable vascular scaffolds. Also, the validation of the tracing methods through the center of the radiopaque marker provides further guidance for the quantitative segmentation of the lumen vessel.

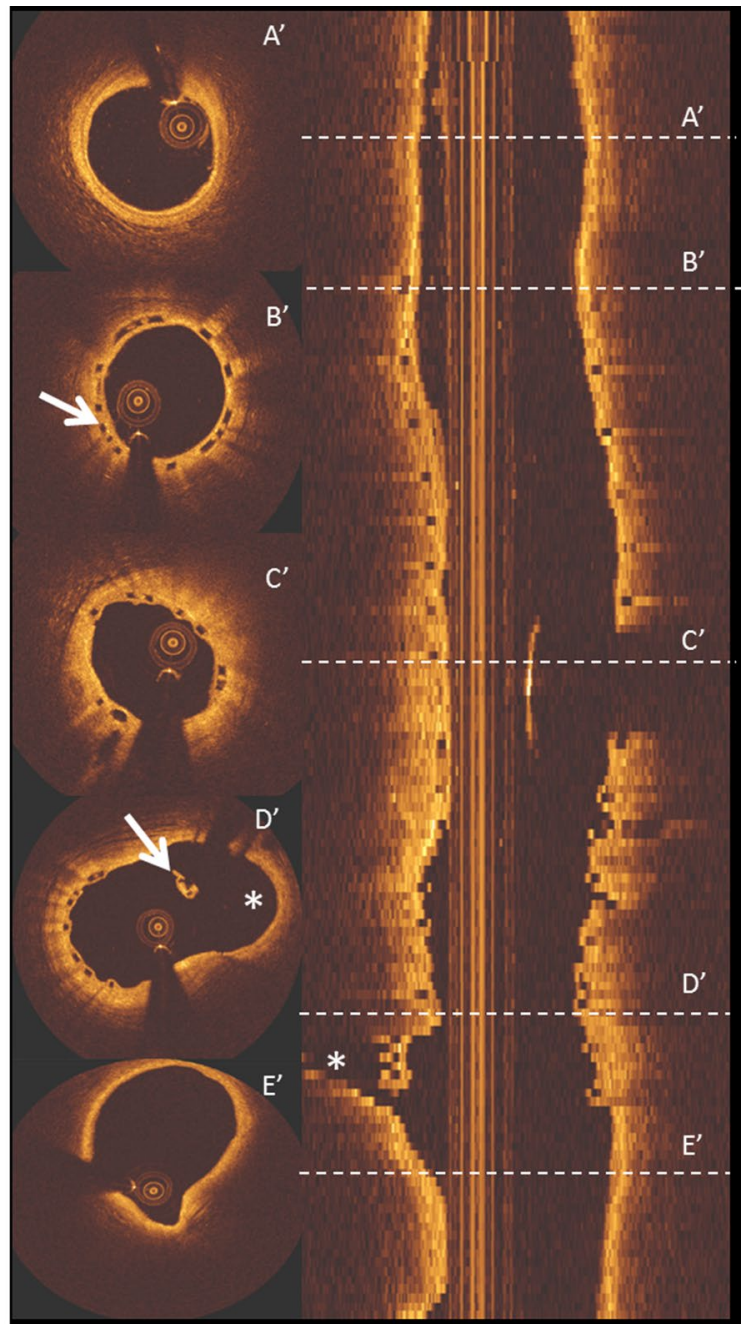

the radiopaque markers, and panel $\mathbf{c}$ and $\mathbf{c}^{\prime}$ correspond to the MLA. The MLA with coronary CTA (c) was $3.4 \mathrm{~mm}^{2}$ and with $4.0 \mathrm{~mm}^{2}$ with OCT $\left(\mathbf{c}^{\prime}\right)$. The markers are shown with white arrows and a white stars identify the sidebranch. CTA computed tomography angiography, MLA Minimal lumen area

To our knowledge this is the first study comparing coronary CTA in-scaffold luminal area dimensions with OCT. The small bias in the assessment of a clinically relevant non-ambiguous parameter, such as MLA, supports the use of coronary CTA angiography as an alternative method to quantify non-invasively in-scaffold luminal area and to further processing using computational fluid dynamics [18]. Indeed, non-invasive coronary CTA angiography have proven to the an accurate tool to assess endothelial shear stress and non-invasive fractional flow reserve $[19,20]$. In the NXT Trial (Analysis of Coronary Blood Flow Using CT Angiography: Next Steps), coronary CTA-derived fractional flow reserve demonstrated 

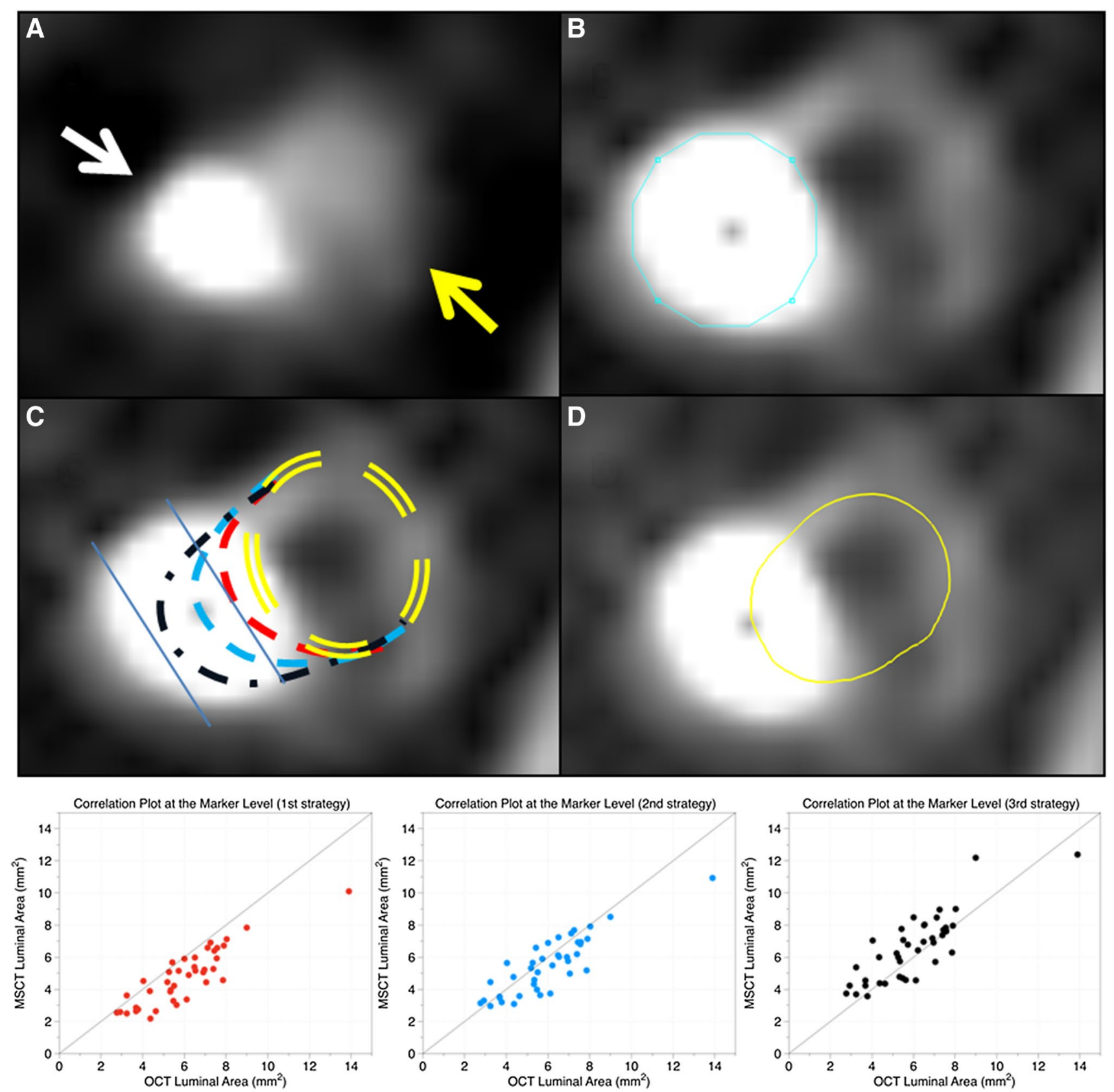

Fig. 4 a Cross-section at the level of the radiopaque marker with the blooming artefact (white arrows) and the coronary artery lumen (yellow arrow). b CT-gradient image at the same cross-section, the radiopaque marker (blue line, area of $2.5 \mathrm{~mm}^{2}$ ) is readily identified. c Three strategies evaluated to measure the agreement between coronary CTA and OCT derived luminal areas (yellow double dash lines represents the position of the lumen with respect to the marker artefact); the first strategy in red dash line included in the luminal area $1.1 \mathrm{~mm}^{2}$ of the blooming artefact without crossing the centre of the

high per-patient sensitivity $86 \%$ (95\% CI 77-92\%) and specificity $79 \%$ (95\% CI 72-84\%) to identify myocardial ischemia compared to invasive FFR [20]. In addition, marker; the second strategy in blue dash line included $2.3 \mathrm{~mm}^{2}$ of the blooming artefact and crossed the centre of the marker and the third strategy in black dash line included $3.2 \mathrm{~mm}^{2}$ of the blooming artefact and the centre of the marker. $\mathbf{d}$ Contour tracing methods with the best agreement (second strategy, blue line) with the OCT-derived matched lumen area. In the bottom, the three tracing strategies are plotted against the OCT-derived lumen area. CTA computed tomography angiography, $O C T$ optical coherence tomography

Bourantas et al. have shown that coronary CTA-derived low shear stress correlates with coronary plaque progression in 3 years of follow up [21]. 


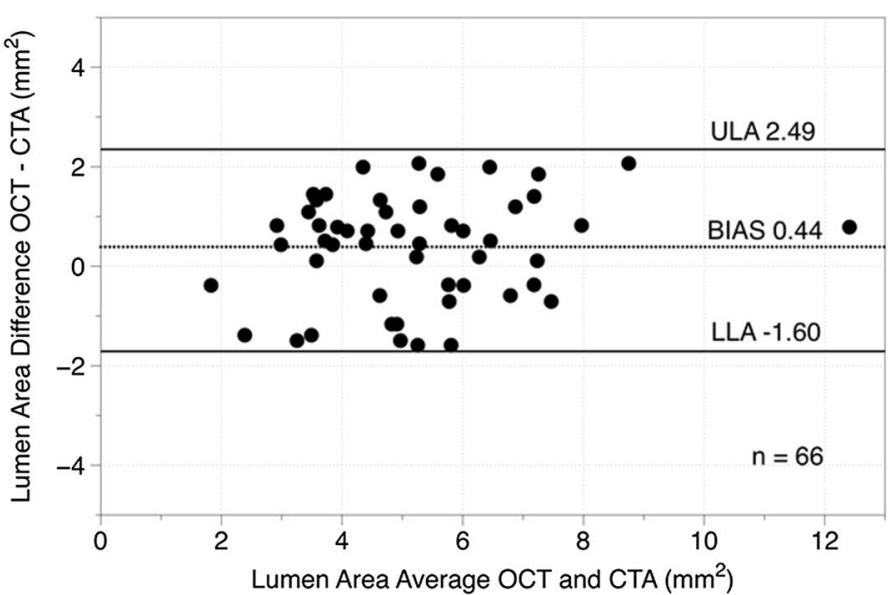

Fig. 5 Bland-Altman scatter plot and Passing-Bablok analysis depicting the global agreement (MLA and radiopaque marker crosssection) between OCT and coronary CTA for quantitative luminal

\section{Limitations}

The main limitation of this study in the inclusion of noncomplex, non-calcified lesion in which coronary CTA has proven to have higher accuracy; therefore, this finding should be confirmed in a more complex population [22]. Also, the low incidence of in-scaffold restenosis limits the conclusion of this analysis to patent scaffolds. Moreover, the $3 \mathrm{~mm}$ scaffold diameter included in this study might have facilitated the quantitative assessment The accuracy of coronary CTA to investigate the commercially available $2.5 \mathrm{~mm}$ diameter scaffold is still unknown. Image acquisition was not performed at the same time; therefore, introducing a confounding factor. Nevertheless, the serial invasive angiography analysis in this population reported stable luminal dimensions in the timeframe evaluated in this study (i.e., after 12 months) [1]. And finally, due to the limitation in spatial resolution of coronary CTA coronary crosssection matched by OCT and coronary CTA may not be exactly identical.

\section{Conclusion}

Compared with OCT, coronary CTA appears to be accurate for the estimation of in-scaffold minimal lumen area 18-month post-implantation, with no difference compared to the non-scaffolded region.

\section{Compliance with ethical standards}

Conflict of interest Y. Onuma and P.W. Serruys are members of International Advisory Board of Abbott Vascular. Y. Sotomi is a consultant for GOODMAN and has received a grant from the Fukuda Memorial Foundation and SUNRISE laboratory. P.H. Kitslaar is an employee

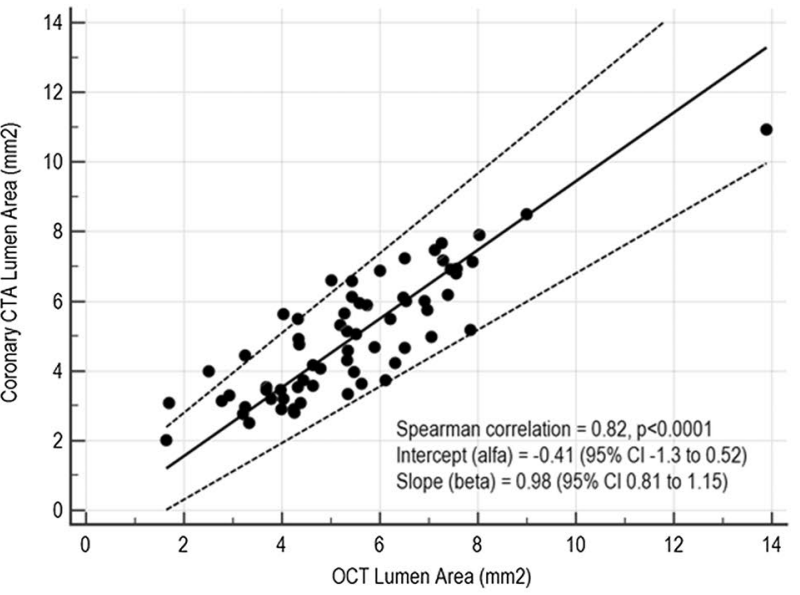

area assessment. CTA computed tomography angiography, MLA minimal lumen area, $O C T$ optical coherence tomography

of Medis medical imaging systems and has a research appointment at the Leiden University Medical Center. All other authors declare no competing interests.

\section{References}

1. Serruys PW, Ormiston J, van Geuns RJ, de Bruyne B, Dudek D, Christiansen E et al (2016) A Polylactide bioresorbable scaffold eluting everolimus for treatment of coronary stenosis: 5-year follow-up. J Am Coll Cardiol 67(7):766-776

2. Stone GW, Gao R, Kimura T, Kereiakes DJ, Ellis SG, Onuma $\mathrm{Y}$ et al (2016) 1-year outcomes with the Absorb bioresorbable scaffold in patients with coronary artery disease: a patient-level, pooled meta-analysis. The Lancet 387(10025):1277-1289

3. Serruys PW, Chevalier B, Dudek D, Cequier A, Carrie D, Iniguez A et al (2015) A bioresorbable everolimus-eluting scaffold versus a metallic everolimus-eluting stent for ischaemic heart disease caused by de-novo native coronary artery lesions (ABSORB II): an interim 1-year analysis of clinical and procedural secondary outcomes from a randomised controlled trial. Lancet 385(9962):43-54

4. Onuma Y, Dudek D, Thuesen L, Webster M, Nieman K, Garcia-Garcia HM et al (2013) Five-year clinical and functional multislice computed tomography angiographic results after coronary implantation of the fully resorbable polymeric everolimus-eluting scaffold in patients with de novo coronary artery disease: the ABSORB cohort A trial. JACC Cardiovasc Interv 6(10):999-1009

5. Gutierrez-Chico JL, Serruys PW, Girasis C, Garg S, Onuma Y, Brugaletta $S$ et al (2012) Quantitative multi-modality imaging analysis of a fully bioresorbable stent: a head-to-head comparison between QCA, IVUS and OCT. Int J Cardiovasc Imaging 28(3):467-478

6. Serruys PW, Onuma Y, Dudek D, Smits PC, Koolen J, Chevalier $B$ et al (2011) Evaluation of the second generation of a bioresorbable everolimus-eluting vascular scaffold for the treatment of de novo coronary artery stenosis: 12-month clinical and imaging outcomes. J Am Coll Cardiol 58(15):1578-1588

7. Nieman K, Serruys PW, Onuma Y, van Geuns RJ, Garcia-Garcia HM, de Bruyne B et al (2013) Multislice computed tomography 
angiography for noninvasive assessment of the 18-month performance of a novel radiolucent bioresorbable vascular scaffolding device: the ABSORB trial (a clinical evaluation of the bioabsorbable everolimus eluting coronary stent system in the treatment of patients with de novo native coronary artery lesions). J Am Coll Cardiol 62(19):1813-1814

8. Zhang YJ, Iqbal J, Nakatani S, Bourantas CV, Campos CM, Ishibashi Y et al (2014) Scaffold and edge vascular response following implantation of everolimus-eluting bioresorbable vascular scaffold: a 3-year serial optical coherence tomography study. JACC Cardiovase Interv 7(12):1361-1369

9. Suwannasom P, Onuma Y, Campos CM, Nakatani S, Ishibashi Y, Tateishi $\mathrm{H}$ et al (2015) Fate of bioresorbable vascular scaffold metallic radio-opaque markers at the site of implantation after bioresorption. JACC Cardiovasc Interv 8(8):1130-1132

10. Onuma Y, Serruys PW (2011) Bioresorbable scaffold: the advent of a new era in percutaneous coronary and peripheral revascularization? Circulation 123(7):779-797

11. Leipsic J, Abbara S, Achenbach S, Cury R, Earls JP, Mancini GJ et al (2014) SCCT guidelines for the interpretation and reporting of coronary CT angiography: a report of the Society of Cardiovascular Computed Tomography Guidelines Committee. J Cardiovasc Comput Tomogr 8(5):342-358

12. Tearney GJ, Regar E, Akasaka T, Adriaenssens T, Barlis P, Bezerra HG et al (2012) Consensus standards for acquisition, measurement, and reporting of intravascular optical coherence tomography studies: a report from the International Working Group for intravascular optical coherence tomography standardization and validation. J Am Coll Cardiol 59(12):1058-1072

13. Bezerra HG, Attizzani GF, Sirbu V, Musumeci G, Lortkipanidze N, Fujino Y et al (2013) Optical coherence tomography versus intravascular ultrasound to evaluate coronary artery disease and percutaneous coronary intervention. JACC Cardiovasc Interv $6(3): 228-236$

14. Altman DG, Bland JM (1986) Comparison of methods of measuring blood pressure. J Epidemiol Community Health 40(3):274-277

15. Arbab-Zadeh A, Miller JM, Rochitte CE, Dewey M, Niinuma H, Gottlieb I et al (2012) Diagnostic accuracy of computed tomography coronary angiography according to pre-test probability of coronary artery disease and severity of coronary arterial calcification. The CORE-64 (coronary artery evaluation using 64-row multidetector computed tomography angiography) International Multicenter Study. J Am Coll Cardiol 59(4):379-387

16. Fischer C, Hulten E, Belur P, Smith R, Voros S, Villines TC (2013) Coronary CT angiography versus intravascular ultrasound for estimation of coronary stenosis and atherosclerotic plaque burden: a meta-analysis. J Cardiovasc Comput Tomogr 7(4):256-266

17. Rief M, Zimmermann E, Stenzel F, Martus P, Stangl K, Greupner J et al (2013) Computed tomography angiography and myocardial computed tomography perfusion in patients with coronary stents: prospective intraindividual comparison with conventional coronary angiography. J Am Coll Cardiol 62(16):1476-1485

18. Sankaran S, Kim HJ, Choi G, Taylor CA (2016) Uncertainty quantification in coronary blood flow simulations: impact of geometry, boundary conditions and blood viscosity. J Biomech 49(12):2540-2547

19. Hetterich H, Jaber A, Gehring M, Curta A, Bamberg F, Filipovic $\mathrm{N}$ et al (2015) Coronary computed tomography angiography based assessment of endothelial shear stress and its association with atherosclerotic plaque distribution in-vivo. PLoS One 10(1): $\mathrm{e} 0115408$

20. Norgaard BL, Leipsic J, Gaur S, Seneviratne S, Ko BS, Ito H, et al (2014) Diagnostic performance of noninvasive fractional flow reserve derived from coronary computed tomography angiography in suspected coronary artery disease: the NXT trial (analysis of coronary blood flow using CT angiography: next steps). J Am Coll Cardiol 63(12):1145-1155

21. Bourantas CV, Papadopoulou SL, Serruys PW, Sakellarios A, Kitslaar PH, Bizopoulos P et al (2015) Noninvasive prediction of atherosclerotic progression: the PROSPECT-MSCT Study. JACC Cardiovasc Imaging 9(8):1009-1011

22. Toepker M, Schlett CL, Irlbeck T, Mahabadi AA, Bamberg F, Leidecker C et al (2010) Accuracy of dual-source computed tomography in quantitative assessment of low density coronary stenosis: a motion phantom study. Eur Radiol 20(3):542-548 